\title{
Parameters Estimation Approach for the MEA/hiPSC-CM Asaays
}

\author{
Julien Bouyssier ${ }^{1,2,3}$, Nejib Zemzemi ${ }^{1,2,3}$ \\ ${ }^{1}$ Inria Bordeaux Sud-Ouest. Carmen team, Talence, France. \\ ${ }^{2}$ IHU LIRYC, Electrophysiology and Heart Modeling Institute, Pessac, France. \\ ${ }^{3}$ IMB UMR CNRS 5251, Talence, France.
}

\begin{abstract}
We propose a mathematical approach for the analysis of drugs effects on the electrical activity of human induced pluripotent stem cell-derived cardiomyocytes (hiPSCCMs) based on multi-electrode array (MEA) experiments. Our goal is to produce an in silico tool able to simulate drugs action in MEA/hiPSC-CM assays and to fit the drug model parameters to the experimental data. The electrical activity of the stem cells at the ion-channel level is modeled using the Paci et al. (2013) transmembrane potential model. We use the bidomain model in order to describe the propagation of the electrical wave in the stem cells preparation. The field potential (FP) measured by the MEA is modeled by the extracellular potential of the bidomain equations. First, we propose a strategy allowing to generate FPs in good agreement with the experimental data. Second, we introduce a drug/ion channels interaction based on a pore block model.

Results show that the model reflects properly the main effects of the drug on the FP. In order to estimate the parameters of the drug model, we define a cost function minimizing the gap between the model and the observed FPs. We use an optimization algorithm based on a gradient descent method where the cost function gradient is computed using an adjoint approach. We generated field potential for the five drugs with fixed gold standard IC50 and drug dose values. Then, supposing that one of the gold standard parameters is not known and adding $10 \%$ gaussian noise, the algorithm is able to estimate this parameter with more than $95 \%$ of accuracy. This approach could also be used in the future to optimize drug doses in order to achieve desired therapeutic effects.
\end{abstract}

\section{INTRODUCTION}

The capability of stem cells to differentiate into different tissue types have been shown in [1]. Human induced pluripotent stem cells (hiPSCs) are considered in this work. These cells are derived by reprogramming somatic cells and can be cultivated in the pluripotency state or differentiated into somatic cell types, including CMs [2]. Until now, the knowledge regarding the cardiac tissue mainly relied on animal models, like dog, rabbit or guinea pig. Today, many types of human pluripotent stem cells are investigated for their potential to produce functional CMs [3], [4]. hiPSC$\mathrm{CMs}$ are valuable models because of their resemblance to adult myocytes, especially in the electrophysiological behavior [5]. Therefore, the role of hiPSC-CMs as in vitro models is becoming more and more important. In hiPSCCMs preparations, a strong heterogeneity in the morphology of the action potentials is usually observed. However, the signals can be classified into three major types: nodal-like, embryonic atrial-like, and embryonic ventricular-like [3], [2].
The hiPSC-CMs have been used in different fields: toxicity testing, study of certain diseases, pharmacological response, drug design etc. [4], [2], [6], [7], [8], [9]. The present study is related to the problem of drug screening in safety pharmacology. Our goal is to model and simulate MEA measurements that are performed by pharmaceutical companies on hiPSCCMs preparations. The aim of these experiments is to predict and test the main effects of a drug on the electrophysiology of cardiomyocytes. But the electrical signal collected by an MEA device, called the Field Potential (FP), is difficult to analyze, because of its variability, and because it has been much less studied than the Action Potential (AP). With this study, we want to show that mathematical modeling and numerical simulation can contribute to a better understanding of the MEA measurements. Some preliminary simulations of the FP were recently presented [10], [11], [12] and the modeling of drug effects, side effects and interactions was addressed in several works [13], [14], [15]. But to the best of our knowledge, the present article is the first in silico study of drug effects on the FP.

Here is a brief description of the methods adopted in this work. Our mathematical model is based on the bidomain equations, used in many works for the description of the electrical activity of the heart. In order to reproduce the electrical activity of hiPSC-CMs, a state-of-the-art ionic model describing the membrane activity of stem cells is used in Paci et al [16]. Since the experimental measurements are registered by MEA devices, a model of electrodes is introduced and coupled to the bidomain equations. The resulting equations allow us to model the field potential recorded by the MEA device. A specific device is considered (the 60-6 well MEA produced by the company Multi Channel Systems) but the methodology can be applied to other kinds of MEAs. This device is made of six independent wells. Each well contains nine electrodes. Six independent experiments can thus be done with identical surrounding conditions at once [17]. The geometry used for the computation is twodimensional. It models one layer of cells in a well, as represented in Fig. 1(a). A special effort is dedicated to the modeling of the electrophysiological heterogeneity, which is a prominent characteristic of hiPSC-CMs preparations. This is done by introducing different phenotypes, atrialand ventricular-like, and by varying the action potential amplitudes. Various configurations are generated following this approach. The in silico results corresponding to the 
different configurations are averaged and compared to in vitro experiments for five different drugs (mexiletine, dofetilide, bepridil, ivabradine and BayK).

Human induced pluripotent stem cell-derived cardiomyocytes are a promising tool in regenerative medicine (repair damaged areas) because of pluripotency and ability to differentiate. Computational modeling and simulation is a powerful tool to investigate [13]:

- drug effects and their side effects

- disease in cardiac electrophysiological activity.

Our goal is to perform in silico simulations (quantify and predict affinities and effects of drugs on hIPSC-CMs) to be used in early stage of the development of new compounds. Here we provide an approach allowing to fit the drug model parameters to the experimental data.

\section{Methods}

\section{A. Forward problem}

In order to model a layer of hIPSC-CMs, we use the bidomain model that we solve in a $2 \mathrm{D}$ computational domain $\Omega$ of Fig. 1(a). We compute the membrane potential $V_{M}$ and the extracellular potential $u_{e}$.

$$
\left\{\begin{array}{l}
\frac{d \mathbf{w}}{d t}-\mathbf{g}\left(V_{M}, \mathbf{w}\right)=0 \text { in } \Omega \\
A_{M}\left(C_{M} \frac{\partial V_{M}}{\partial t}+I_{\text {ion }}\right)-\operatorname{div}\left(\sigma_{I} \nabla V_{M}\right)-\operatorname{div}\left(\sigma_{E} \nabla u_{e}\right)=A_{M} I_{s t i m} \text { in } \Omega \\
-\operatorname{div}\left(\left(\sigma_{I}+\sigma_{E}\right) \nabla u_{e}\right)-\operatorname{div}\left(\sigma_{I} \nabla V_{M}\right)=\frac{1}{z_{\text {thick }}} \Sigma_{e_{k}} \frac{I_{e l}^{k}}{\left|e_{k}\right|} \chi_{e_{k}} \text { in } \Omega,
\end{array}\right.
$$

where $\left|e_{k}\right|$ denotes the surface of the electrode $k$, and $\chi_{e_{k}}$ denotes its characteristic function, i.e. the function equal to one inside the electrode and zero outside. Functions $V_{M}$ and $u_{e}$ are respectively the transmembrane and extracellular potentials. The constant $C_{M}$ is the transmembrane specific capacitance, $A_{M}$ is the surface area of membrane per unit volume of tissue, $\sigma_{I}$ and $\sigma_{E}$ are respectively the intracellular and extracellular conductivities. The transmembrane ionic current $I_{i o n}$ is provided by the model proposed in Paci et al [16]. The electrodes are described using the circuit in

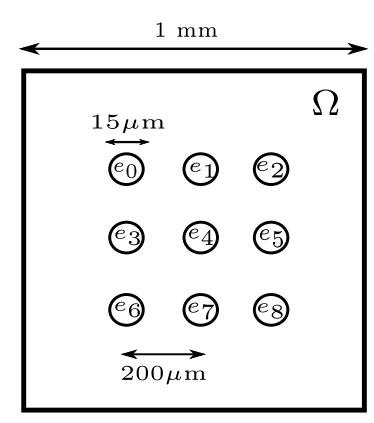

(a) Domain

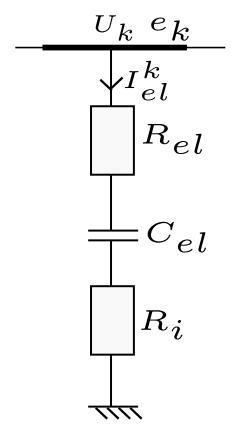

(b) Electrodes circuit
Fig. 1. Schematic representation of the electrodes electrical circuit (a). $2 \mathrm{D}$ domain $\Omega$ with dimensions and positions of the 9 electrodes (b) Computational mesh representing a triangular discretization of the domain $\Omega$ with $h \approx 25 \mu \mathrm{m}$ (c).

Fig. 1(b), by computing the measured current $I_{e l}^{k}$ : by solving the following ODE:

$$
\frac{d I_{e l}^{k}}{d t}+\frac{I_{e l}^{k}}{\tau}=\frac{C_{e l}}{\tau} \frac{d U^{k}}{d t} \quad \text { with } U^{k}=\frac{1}{\left|e_{k}\right|} \int_{e_{k}} u_{e} d e_{k},
$$

where $\tau=\left(R_{i}+R_{e l}\right) C_{e l}, R_{i}$ standing for the ground resistance, $R_{e l}$ and $C_{e l}$ for the resistance and the capacitance of the electrode. In Eq. (2) we have introduced $U^{k}$ as the mean value of the extracellular potential $u_{e}$ over the electrode $k$. The measured field potential is then $U_{\text {meas }}^{k}=R_{i} I_{e l}^{k}$.

\section{B. Model for the drug/ion channels interactions}

The in silico approach presented in the previous sections was designed to qualitatively mimic the field potential acquired with MEA measurements, by taking into account a certain amount of variability. Our purpose is now to introduce in the model the action of compounds on ion channels. The modeling of drug-channel interactions has been the object of several studies (see e.g. [14], [18]). Various concepts have been introduced 1) pore block action: the flow of ions is inhibited by the drug binding with a continuously accessible channel receptor; 2) modulated and guarded receptor theories: the drug access to the binding sites is restricted due to the channel conformation during the AP cycle; 3) allosteric effectors: a drug binding to a protein changes its activity and also activates conformational changes in its dynamics. In the present study, the pore block model is used because it does not require too many parameters and because it proved to be able to reproduce the relevant phenomena. The pore block model is implemented with the "conductanceblock" formulation [19], [20], [13], [21]. The conductance of the targeted channel is reduced by a scaling factor in the following way:

$$
g_{s}=g_{\text {control }, s}\left[1+\left(\frac{[D]}{I C_{50}}\right)^{n}\right]^{-1}
$$

where $g_{\text {control,s }}$ is the drug-free maximal conductance of channel $s$, the $I C_{50}$ value of the drug is the drug concentration at which a $50 \%$ reduction of the specific channel peak current is observed and $[D]$ is the drug concentration. The Hill coefficient $n$ will be assumed to be equal to 1 .

\section{Optimization strategy}

For given synthetical field potential measurements $U_{\text {meas }}^{k}$ where a drug is blocking one ionic channel, our goal is to find the dose $[D]_{\text {opt }}$ allowing to obtain these data and knowing the concerned drug and its targeted channel.

First, we define a cost function $J$ depending on the drug dose $[D]$ minimizing the gap between the simulated and the field potential measurements:

$$
J([D])=\frac{1}{2} \Sigma_{e_{k}}\left\|R_{i} I_{e l}^{k}([D])-U_{\text {meas }}^{k}\right\|_{l^{2}([0, T])}^{2}
$$

where $I_{e l}^{k}([D])$ is solution of the tissue model ((1)-(2)). Our goal is to find $[D]$ minimizing (4). For this, we define an adjoint problem associated to the bidomain coupled to the 
measurement system model

$$
\left\{\begin{array}{c}
\frac{d \mathbf{q}}{d t}+{ }^{t}\left(\partial_{\mathbf{w}} \mathbf{g}\left(V_{M}, \mathbf{w},[D]\right)\right) \mathbf{q}-A_{M}{ }^{t}\left(\partial_{\mathbf{w}} I_{\text {ion }}\left(V_{M}, \mathbf{w},[D]\right)\right) p_{M}=0 \text { in } \Omega, \\
A_{M}\left(C_{M} \frac{\partial p_{M}}{\partial t}-\partial_{V_{M}} I_{i o n}\left(V_{M}, \mathbf{w},[D]\right)\right) p_{M}+\operatorname{div}\left(\sigma_{I} \nabla p_{M}\right) \\
\quad+\operatorname{div}\left(\sigma_{I} \nabla p_{e}\right)+{ }^{t}\left(\partial_{V_{M}} \mathbf{g}\left(V_{M}, \mathbf{w},[D]\right)\right) \mathbf{q}=0 \text { in } \Omega \\
\operatorname{div}\left(\left(\sigma_{I}+\sigma_{E}\right) \nabla p_{e}\right)+\operatorname{div}\left(\sigma_{I} \nabla p_{M}\right)=\Sigma_{e_{k}} \frac{C_{e l}}{\tau} \frac{1}{\left|e_{k}\right|} \frac{d \lambda^{k}}{d t} \chi_{e_{k}} \text { in } \Omega, \\
\frac{d \lambda^{k}}{d t}-\frac{\lambda^{k}}{\tau}=-\frac{1}{z_{\text {thick }}} P_{e}^{k}-R_{i}\left(R_{i} I_{e l}^{k}-U_{m}^{k}\right) \text { in } e_{k}, \text { for } k=0, . ., 8 .
\end{array}\right.
$$

where $\mathbf{q}, p_{M}, p_{e}$ and $\lambda^{k}$ are the adjoint states associated respectively to variables $\mathbf{w}, V_{M}, u_{e}$ and $I_{e l}^{k}$ and solution of (5). The variable $P_{e}^{k}$ is given by $P_{e}^{k}=\frac{1}{\left|e_{k}\right|} \int_{e_{k}} p_{e} d e^{k}$. In order to estimate the parameters of the drug model, we use an optimization procedure based on a gradient descent method where the cost function gradient is computed using the state and adjoint state variables as follows

$$
\begin{array}{r}
\partial_{[D]} J([D])=\int_{0}^{T} \int_{\Omega} t^{t}\left(\partial_{[D]} \mathbf{g}\left(\mathbf{V}_{\mathbf{M}}, \mathbf{w},[\mathbf{D}]\right)\right) \mathbf{q} \\
-\int_{0}^{T} \int_{\Omega} A_{M} \partial_{[D]} I_{i o n}\left(V_{M}, \mathbf{w},[D]\right) p_{M} .
\end{array}
$$

Giving an initial guess $[D]_{\text {guess }}$, we solve the optimization problem using the following algorithm. Here, $\epsilon_{\text {Func }}$ and

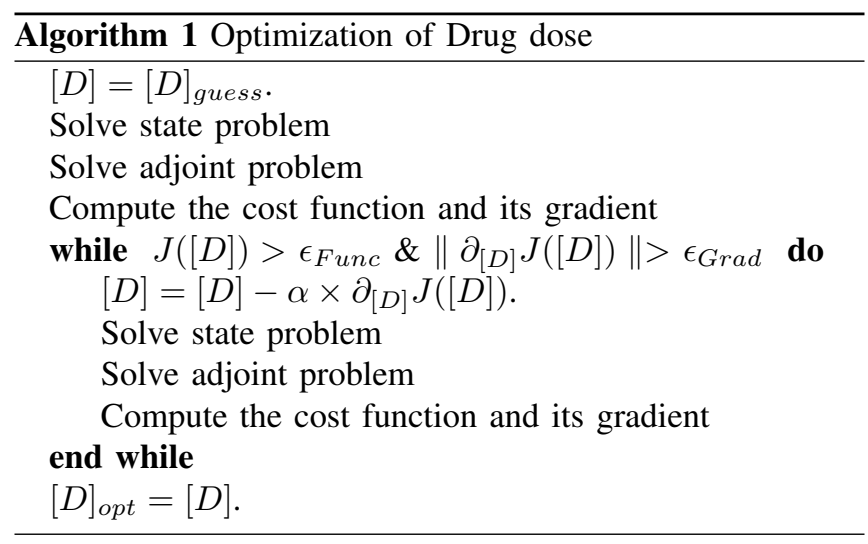

$\epsilon_{\text {Grad }}$ are positive constants defining the desired tolerance on the cost function and its gradient respectively. The coeficient $\alpha$ is positive and could be fixed or updated at each iteration.

\section{RESULTS}

In Fig 2, we show the simulated field potential over the nine electrode in the control condition and when introducing $50 \mu M$ and $100 \mu M$ of mexilitine. we observe that the simulated field potential are in agreement with the experimental field potentials in terms of depolarization and repolarization times but not in terms of magnitude. More experimental data could be found in [22]. In figure 3, we show the robustness of the proposed method with respect of the initial guess $[D]_{\text {guess }}$ and also with respect to noise by adding $5 \%$ and $10 \%$ of noise on the recorded data. In the presence of noise, the cost function seems to stagnate as shown in figures 4 and so is value of [D] as shown in figure 3 . But the obtained dose is $99 \%$ accurate even with $10 \%$ of noise in the recorded data. We provide a snapshot of how the adjoint state associated to the action potential looks like in figure 5. It is not easy to interpret the adjoint states as they do not represent physical quantities. The only thing that we can expect is that they converge to zero when the dose converges to the exact value.

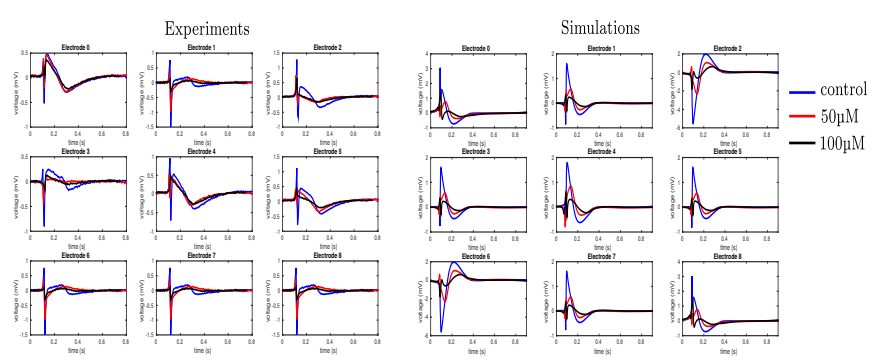

Fig. 2. Example of experimental measurement (left) and simulated (right) field potential. The nine traces are the signals recorded by the nine electrodes as shown in figure1(a)

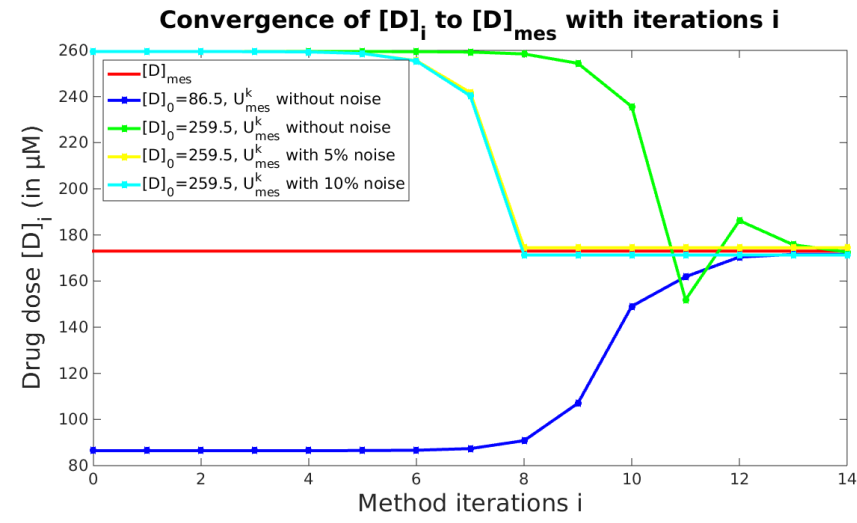

Fig. 3. INa channel block estimation. Convergence of the drug dose starting from different initial guess and with different levels of noise on the observed signals. Relative error is less than $1 \%$ for all cases.

\section{CONCLUSION}

In this paper, we presented a proof of concept of an optimization approach able to estimate the drug dose of a compound when the characteristics of the drug are known. The robustness of the method was tested on drugs targeting $I N a$ channel using in silico experiments: with $10 \%$ of gaussian noise on the observed field potential, the accuracy of the estimated drug dose is higher than 99\%. Future developments would include estimating model parameters of drugs like Mexelitine or Dofetilide, drugs with several targeted channels and fitting of other parameters as $I C_{50}$, etc.

\section{ACKNOWLEDGEMENT}

This work was supported by the Agence Nationale de la Recherche (Grant IHU LIRYC ANR-10-IAHU-04). 


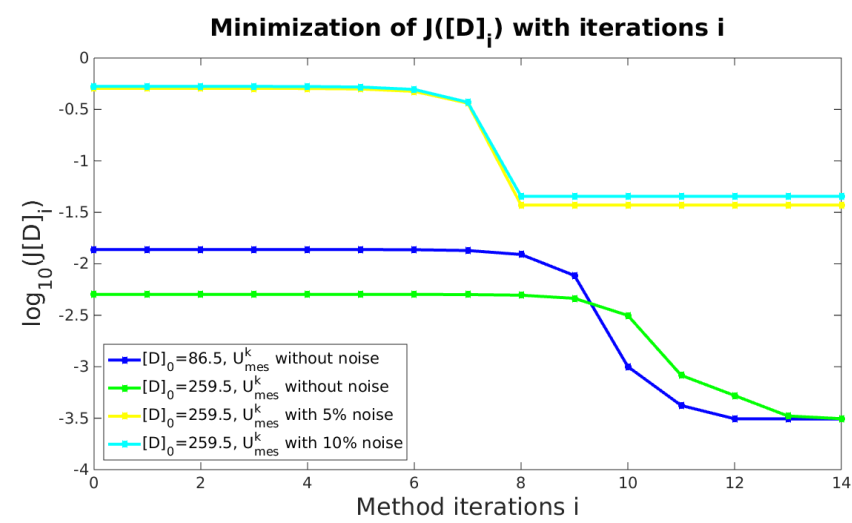

Fig. 4. Convergence of the cost function for different values of $[D]_{\text {guess }}$ and noise levels.

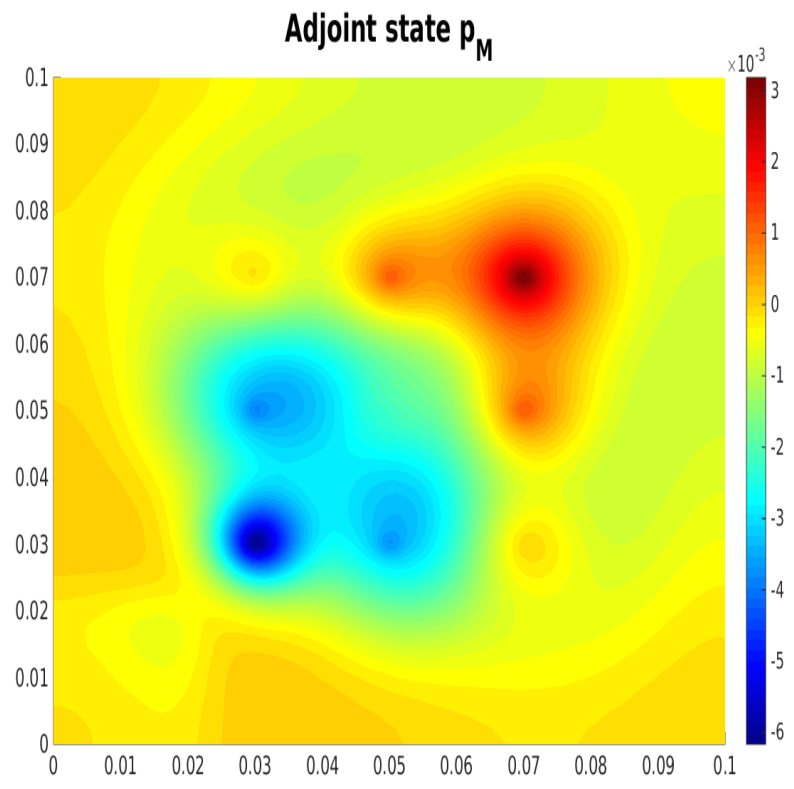

Fig. 5. Snapshot of the propagation of the adjoint state $p_{M}$ used for computing the gradient of the cost function.

\section{REFERENCES}

[1] Takahashi K, Yamanaka S. Induction of pluripotent stem cells from mouse embryonic and adult fibroblast cultures by defined factors. cell 2006;126(4):663-676.

[2] Doss MX, Di Diego JM, Goodrow R, Wu Y, Cordeiro JM, Nesterenko V, Barajas-Martínez H, Hu D, Urrutia J, Desai M, Antzelevitch C. Maximum diastolic potential of human induced pluripotent stem cellderived cardiomyocytes depends critically on ikr. PloS one 2012; 7(7):e40288

[3] He J, Ma Y, Lee Y, Thomson JA, Kamp TJ. Human embryonic stem cells develop into multiple types of cardiac myocytes action potential characterization. Circulation research 2003;93(1):32-39.

[4] Blazeski A, Zhu R, Hunter D, Weinberg S, Boheler K, Zambidis E, Tung L. Electrophysiological and contractile function of cardiomyocytes derived from human embryonic stem cells. Progress in biophysics and molecular biology 2012;110(2):178-195.

[5] Peng S, Lacerda AE, Kirsch GE, Brown AM, Bruening-Wright A. The action potential and comparative pharmacology of stem cell-derived human cardiomyocytes. Journal of pharmacological and toxicological methods 2010;61(3):277-286.

[6] Robertson C, Tran DD, George SC. Concise review: Maturation phases of human pluripotent stem cell-derived cardiomyocytes. Stem Cells 2013;31(5):829-837.

[7] Harris K, Aylott M, Cui Y, Louttit J, McMahon N, Sridhar A. Comparison of electrophysiological data from human-induced pluripotent stem cell-derived cardiomyocytes to functional preclinical safety assays. toxicological sciences $2013 ; \mathrm{kft} 113$.

[8] Braam SR, Tertoolen L, van de Stolpe A, Meyer T, Passier R, Mummery CL. Prediction of drug-induced cardiotoxicity using human embryonic stem cell-derived cardiomyocytes. Stem cell research 2010; $4(2): 107-116$

[9] Caspi O, Itzhaki I, Kehat I, Gepstein A, Arbel G, Huber I, Satin $\mathrm{J}$, Gepstein L. In vitro electrophysiological drug testing using human embryonic stem cell derived cardiomyocytes. Stem cells and development 2009;18(1):161-172.

[10] Bowler L, Harris K, Gavaghan D, Mirams G. Simulated microelectrode array recordings from stem cell-derived cardiomyocytes. Journal of Pharmacological and Toxicological Methods 2016;81:380.

[11] Cavero I, Guillon JM, Ballet V, Clements M, Gerbeau JF, Holzgrefe H. Comprehensive in vitro Proarrhythmia Assay (CiPA): Pending issues for successful validation and implementation. Journal of Pharmacological and Toxicological Methods 2016;URL https:// hal.inria.fr/hal-01328481.

[12] Boulakia M, Raphel F, Zitoun P, Gerbeau JF. Toward transmembrane potential estimation from in vitro multi-electrode field potentials using mathematical modeling. Journal of Pharmacological and Toxicological Methods 2015;75:168-169.

[13] Zemzemi N, Bernabeu M, Saiz J, Cooper J, Pathmanathan P, Mirams G, Pitt-Francis J, Rodriguez B. Computational assessment of druginduced effects on the electrocardiogram: from ion channel to body surface potentials. British journal of pharmacology 2013;168(3):718 733.

[14] Brennan T, Fink M, Rodriguez B. Multiscale modelling of druginduced effects on cardiac electrophysiological activity. European Journal of Pharmaceutical Sciences 2009;36(1):62-77.

[15] Zemzemi N, Rodriguez B. Effects of 1-type calcium channel and human ether-a-go-go related gene blockers on the electrical activity of the human heart: a simulation study. Europace 2015;17(2):326333.

[16] Paci M, Hyttinen J, Aalto-Setälä K, Severi S. Computational models of ventricular-and atrial-like human induced pluripotent stem cell derived cardiomyocytes. Annals of biomedical engineering 2013;41(11):2334 2348

[17] multichannel systems. Microelectrode array (mea) manual. http://www.multichannelsystems.com/sites/ multichannelsystems.com/files/documents/ manuals/MEA_Manual.pdf.

[18] Carmeliet E, Mubagwa K. Antiarrhythmic drugs and cardiac ion channels: mechanisms of action. Progress in biophysics and molecular biology 1998;70(1):1-72.

[19] Mirams G, Davies M, Cui Y, Kohl P, Noble D. Application of cardiac electrophysiology simulations to pro-arrhythmic safety testing. British journal of pharmacology 2012;167(5):932-945.

[20] Mirams G, Cui Y, Sher A, Fink M, Cooper J, Heath B, McMahon N, Gavaghan D, Noble D. Simulation of multiple ion channel block provides improved early prediction of compounds clinical torsadogenic risk. Cardiovascular research 2011;91(1):53-61.

[21] Bottino D, Penland RC, Stamps A, Traebert M, Dumotier B, Georgieva A, Helmlinger G, Lett GS. Preclinical cardiac safety assessment of pharmaceutical compounds using an integrated systems-based computer model of the heart. Progress in biophysics and molecular biology 2006;90(1):414-443.

[22] Abbate E, Boulakia M, Coudière Y, Gerbeau JF, Zitoun P, Zemzemi $\mathrm{N}$. In silico assessment of the effects of various compounds in MEA/hiPSC-CM assays: Modelling and numerical simulations. Research report, Inria, July 2017. URL https://hal.inria.fr/ hal-01562673.

Contact: Nejib Zemzemi, 200 avenue de la vieille tour 33405, Talence, France. Email: nejib.zemzemi@ inria.fr

Julien Bouyssier, 200 avenue de la vieille tour 33405, Talence, France. Email: julien.bouyssiereinria.fr 